\title{
The J-Domain of Heat Shock Protein 40 Can Enhance the Transduction Efficiency of Arginine-Rich Cell-Penetrating Peptides
}

\author{
Tzu-Yin Lin, ${ }^{1}$ Yu-Hsiu Su, ${ }^{1}$ Kun-Hsiung Lee, ${ }^{2}$ and Chin-Kai Chuang ${ }^{1}$ \\ ${ }^{1}$ Division of Animal Technology, Animal Technology Laboratories, Agricultural Technology Research Institute, No. 1, Lane 51, \\ Dahu Road, Xiangshan District, Hsinchu 30093, Taiwan \\ ${ }^{2}$ Division of Biotechnology, Animal Technology Institute Taiwan, No. 52, Kedong 2nd Road, Chunan, Miaoli 35059, Taiwan
}

Correspondence should be addressed to Chin-Kai Chuang; jkjuang@mail.atit.org.tw

Received 1 September 2014; Revised 28 November 2014; Accepted 16 December 2014

Academic Editor: Alvaro R. Lara

Copyright (C) 2015 Tzu-Yin Lin et al. This is an open access article distributed under the Creative Commons Attribution License, which permits unrestricted use, distribution, and reproduction in any medium, provided the original work is properly cited.

\begin{abstract}
Sense and antisense oligonucleotide pairs encoding cell-penetrating peptides PTD ( Tat $_{47-57}$ ), DPV3A, E162, pVEC, R11, and TP13 were used to construct two sets of pET22b-CPP-DsRed and pET22b-CPP-J-DsRed vectors for CPP-DsRed and CPP-J-DsRed recombinant proteins expression. PTD-DsRed, DPV3A-DsRed, PTD-J-DsRed, and DPV3A-J-DsRed recombinant proteins were expressed in a soluble form. PTD-J-DsRed and DPV3A-J-DsRed recombinant proteins were able to escape from E. coli host cells into the culture medium. The membrane-penetrating activity of PTD-J-DsRed and DPV3A-J-DsRed recombinant proteins to mammalian cells was more effective than that of PTD-DsRed and DPV3A-DsRed. The route of the cellular membrane translocation of these recombinant proteins is suggested via macropinocytosis followed by an endosomal escape pathway.
\end{abstract}

\section{Introduction}

1.1. Cell-Penetrating Peptides (CPPS). Since the observation that HIV-1 Tat protein could shuttle between cells and the discovery that purified Tat protein could enter cells and translocate into nuclei [1], the cell-penetrating activity of Tat has been narrowed down gradually from amino acids 36-72 [2], to either amino acids 48-60 [3] or amino acids 47-57 [4]. The protein transduction domain (PTD, specifically indicating the peptide: Tat amino acids $47-57$, hereafter) of Tat protein was able to deliver macromolecule, such as $120 \mathrm{kDa} \beta$-galactosidase, fused to it in vivo $[5,6]$. Meanwhile, a 16-amino acid peptide derived from the third helix of the homeodomain of Antennapedia, termed as penetratin, was found to translocate through cell membrane as well [7]. Up to now, a lot of cellpenetrating peptides (CPPs) have been reported (for review, see [8]) and the information has been collected and compiled in a website [9]. CPPs, either protein-derived or chemically synthesized, can be categorized into primary amphipathic, secondary amphipathic, and nonamphipathic [10]. The primary amphipathic CPPs such as transportan [11] and TP10 [12] are usually longer than 20 amino acids with periodically hydrophobic and hydrophilic residues along the primary sequence. In comparison to the primary amphipathic CPPs, the secondary amphipathic CPPs such as penetratin, pVEC [13], and E162 [14] contain less amino acid residues and perform amphipathic structure upon interacting with phospholipid membrane. The third class CPPs, such as R8 [15], DPV3 [16], and PTD [4], are relatively short and contain very high content of arginine.

1.2. Membrane-Penetration Mechanisms of Arginine-Rich CPPs. A metabolic energy-independent, direct plasma membrane translocation mechanism could be detected for the highly positively charged $\mathrm{R} 8$ peptide at $4^{\circ} \mathrm{C}$ at which the receptor-mediated internalization was completely inhibited. However, only a small part of R8 penetration occurred by way 
of this pathway at physiological temperature [17]. Pyrenebutyrate can neutralize the positive charge of R8 [15], R9, and Tat $_{48-60}[18]$ and provide a hydrophobic aromatic group to accelerate the direct penetration through the cellular membrane. At $37^{\circ} \mathrm{C}$, the translocation of arginine-rich CPPs into cytoplasm is mainly via an endocytic uptake-endosomal escape pathway. The translocation of arginine-rich CPPs is not dependent on both clathrin and caveolin-coated pitmediated endocytosis but is inhibited by 5 -(N-ethyl-N-isopropyl)amiloride (EIPA), an inhibitor of macropinocytosis [19], and cytochalasin D, which prevents actin polymerization [20]. Membrane-associated proteoglycan including heparin sulfate (HSPG) is reported to play a crucial role in the endocytic uptake of arginine-rich CPPs [21]. It could be concluded that the positively charged arginine-rich CPPs associated with negatively charged proteoglycan on the surface of plasma membrane were engulfed by macropinocytosis followed by endosomal escape into the cytoplasm. The endosomal escape step is rate limiting for the CPPs to arrive at the cytosol; however, the mechanism is not well explored.

1.3. PTD-J-Domain. Recently, a vector $\mathrm{pET} 22 \mathrm{~b}-\mathrm{PTD}_{1} \mathrm{~J}_{1}$ that could be used to highly express recombinant protein fused to PTD-J-domain on its N-terminus was reported. We took advantages of the specific association ability of the J-domain of Hsp40 with the nucleotide binding domain of Hsp70 and the cell membrane-penetrating activity of the protein transduction domain of HIV-1 Tat protein. Higher level and more soluble chicken IGF-I recombinant protein was expressed by the $\mathrm{pET} 22 \mathrm{~b}-\mathrm{PTD}_{1} \mathrm{~J}_{1}$ vector in comparison to the $\mathrm{pET} 32 \mathrm{~b}$ vector. An HpNC peptide containing two fragments of human heptoprotein was expressed by the pET22b-PTD $\mathrm{J}_{1}$ vector. The PTD-J-HpNC recombinant polypeptide product could effectively elicit rat antisera specific to subtypes $\mathrm{Hp} 1$ and $\mathrm{Hp} 2$ heptoproteins in human serum samples, but the counterpart TrxA-HpNC could not [22]. Moreover, overexpression of PTD-J-FMDVepi, where FMDVepi is an assembled $\mathrm{T}_{\mathrm{H}}$ and B-epitopes of foot-and-mouth disease virus VP1 capsid protein, is dependent on the combination of PTD and Jdomain rather than PTD or J-domain individually. This result suggests that the fused PTD-J polypeptide may possess a special structure that can elicit the immunogenicity of FMDVepi peptide fused with it [23].

In this study, two sets of pET22b-CPP and pET22b-CPPJ expression vectors were constructed. The CPP-DsRed and CPP-J-DsRed recombinant proteins expressed by them were characterized. The cellular membrane-penetrating capabilities of the chosen CPPs were elevated by the J-domain fused to them.

\section{Materials and Methods}

2.1. Construction of pET22b-CPP-DsRed and pET22b-CPP-JDsRed Vectors. The coding region of the red fluorescence protein in pDsRed monomer N1 plasmid (Cat. no. 632465, Clontech) was amplified by PCR with forward primer (G AAT TCT CAT ATG ATG GAC AAC ACC GAG GAC GTC ATC) and reverse primer (CTC GAG ACC ACC CTG GGA GCC GGA GTG GCG GGC CT). The amplified
DNA fragment was ligated with pGEM TEasy TA-cloning vector (A1360, Promega) to get pGEM TE-DsRed. Then, the cloned DsRed DNA fragment was removed from the cloning vector by EcoRI and XhoI restriction enzyme digestion and subcloned to the pET22b-PTD $\mathrm{J}_{1}$ expression vector [22] which had been treated by the same pair of restriction enzymes to obtain pET22b-PTD-J-DsRed plasmid. The sense and antisense oligonucleotide pairs of PTD, DPV3A (the first amino acid residue $\mathrm{R}$ of DPV3 was replaced by $\mathrm{A}$ ), E162, pVEC, R11, and TP13 (Table 1) were annealed in TEN (10 mM Tris-HCl, pH 8.0/1 mM EDTA, pH 8.0/0.3 M NaCl) at $60^{\circ} \mathrm{C}$ for $30 \mathrm{~min}$ followed by slowly cooling down to room temperature for about $60 \mathrm{~min}$. These annealed primer pairs were inserted between NdeI and EcoRI sites of pET22b to obtain pET22b-PTD, pET22b-DPV3A, pET22b-E162, pET22b-pVEC3, pET22b-R11, and pET22b-TP13. The DsRed DNA fragment described above was inserted between the EcoRI and XhoI sites of pET22b-PTD, pET22b-DPV3A, pET22b-E162, pET22b-pVEC3, pET22b-R11, and pET22bTP13 to get pET22b-PTD-DsRed, pET22b-DPV3A-DsRed, pET22b-E162-DsRed, pET22b-pVEC3-DsRed, pET22b-R11DsRed, and pET22b-TP13-DsRed, respectively. The J-DsRed fragment removed from pET22b-PTD-J-DsRed by BamHI and XhoI codigestion was inserted between BamHI and XhoI sites of pET22b-DPV3A, pET22b-E162, pET22b-pVEC3, pET22b-R11, and pET22b-TP13 to get pET22b-DPV3AJ-DsRed, pET22b-E162-J-DsRed, pET22b-pVEC3-J-DsRed, pET22b-R11-J-DsRed, and pET22b-TP13-J-DsRed, respectively.

2.2. Construction of pET22b-DsRed and pET22b-J-DsRed Vectors. The $0.7 \mathrm{~kb}$ NdeI-XhoI fragment of pGEM TE-DsRed clone was inserted into the same restriction enzyme sites of pET22b to obtain pET22b-DsRed. The primer pair, CAT ATG GGT AAA GAT TAC TAC CAG ACT CAC GGT and GA ATT CGA ACC ACG TGG AAC TAA ATT CGC ACC ACC AGA, was used to amplify DNA fragment encoding the J-domain and a thrombin cutting site using $\mathrm{pET} 22 \mathrm{~b}-\mathrm{PTD}_{1} \mathrm{~J}_{1}$ as template. This DNA fragment was utilized to replace the PTD-J fragment which is franked by NdeI and EcoRI sites of the pET22b-PTD $\mathrm{J}_{1}$-DsRed vector to prepare pET22b-JDsRed.

\subsection{Expression and Purification of CPP-DsRed, CPP-J-DsRed,} DsRed, and J-DsRed Recombinant Proteins. The E. coli Rosetta gamiB(DE3)pLysS host cells transformed by pET22b-CPPDsRed, pET22b-CPP-J-DsRed, pET22b-DsRed, or pET22bJ-DsRed were grown in $2 \mathrm{x}$ YT supplemented with $0.4 \%$ glucose, $30 \mu \mathrm{g} / \mathrm{mL}$ chloramphenicol, and $50 \mu \mathrm{g} / \mathrm{mL}$ ampicillin at $37^{\circ} \mathrm{C}$. IPTG was adjusted to $1 \mathrm{mM}$ when $\mathrm{OD}_{600}$ was 0.6 and cells were cultured for another $4 \mathrm{~h}$. To analyze recombinant proteins released into the medium, cells were centrifuged at $12,000 \mathrm{~g}$ for $30 \mathrm{~min}$. The supernatant was concentrated 10 -fold using Centricon (Y3, Millipore), and then $30 \mu \mathrm{L}$ of sample was loaded in each lane of a 12\% SDS polyacrylamide gel. To analyze recombinant proteins within cells, the cells were collected by centrifugation at $10,000 \mathrm{~g}$ for $10 \mathrm{~min}$. After ultrasonication, protein contents of soluble fraction and insoluble 


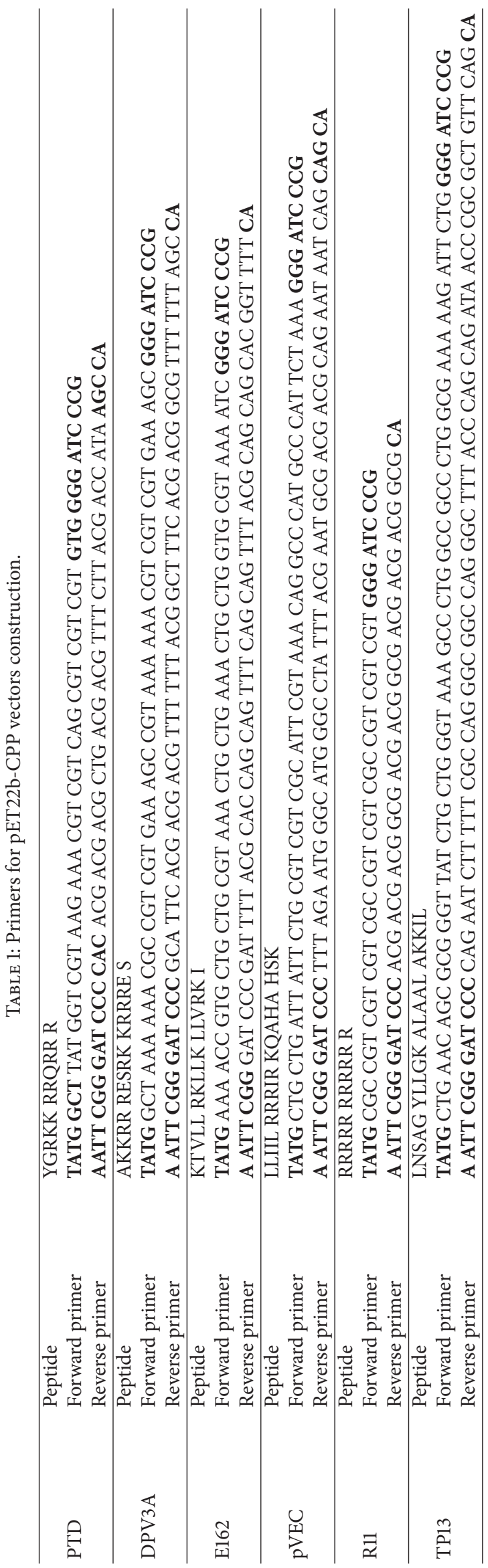


fraction corresponding to $0.2 \mathrm{OD}_{600}$ unit of cells were run on a $12 \%$ SDS polyacrylamide gel. The gel was stained with Coomassie Blue R250. Soluble forms of CPP-DsRed and CPPJ-DsRed recombinant proteins were purified by Ni-Sepharose 6 Fast Flow affinity column (17-5318-02, GE) in accordance with the manufacture's instruction.

2.4. Protein Transduction. Huh-7 cells were seeded at $1.5 \times 10^{5}$ cells per well in a 24-well plate and cultured in DMEM supplemented with $10 \%$ FBS the day before protein transduction experiment. The cells were washed with serum-free medium twice and incubated with various concentrations of recombinant proteins in serum-free medium for various times as indicated. The unpenetrated recombinant proteins were washed off with PBS twice. Then, the penetrated proteins were released from cells using $200 \mu \mathrm{L}$ of PBS supplemented with $1 \%$ Triton X-100. After centrifugation at $10,000 \mathrm{~g}$ for $5 \mathrm{~min}$ to remove the nonsoluble materials, $100 \mu \mathrm{L}$ supernatant was transferred to a well of a 96-well plate to measure the amount of recombinant DsRed proteins in the soluble fraction using a fluorometer. The stimulating wave length and emission wave length were set at $557 \mathrm{~nm}$ and $585 \mathrm{~nm}$, respectively. Experiments were repeated for four times. The recombinant DsRed proteins penetrated into cells were also detected with a fluorescence microscope. The calibration curves between the fluorescence values of $100 \mu \mathrm{L}$ sample per well and the concentrations of recombinant proteins in total nontransduced cell lysate were measured as described above. To test the effects of endocytosis inhibitors on the cell-penetration activity of PTD-J-DsRed, cells were pretreated with filipin $(5 \mu \mathrm{g} / \mathrm{mL}$; Sigma, F9765), EIPA (100 $\mu$ M; Sigma, A3085), or cytochalasin $\mathrm{D}(10 \mu \mathrm{M}$; Sigma, C8273) for 1 hour before the treatment of the PTD-J-DsRed recombinant protein $(40 \mu \mathrm{g} / \mathrm{mL})$ for two hours.

\section{Results}

3.1. Construction of pET22b-CPP-DsRed and pET22b-CPP-JDsRed Expression Vectors. Published CPPs with better transduction activities were focused and TP13 (primary amphipathic CPP) [24], E162, and pVEC (secondary amphipathic CPPs) [14], as well as R11 [25], PTD [22], and DPV3 [16] (arginine-rich CPPs), were picked up in this study. The pET22b-CPP-DsRed and pET22b-CPP-J-DsRed expression vectors were constructed as described in Section 2 and the representative structures of CPP-DsRed and CPP-J-DsRed accompanied with the DsRed and J-DsRed controls are shown in Figure 1.

3.2. Expression of CPP-DsRed Recombinant Proteins. After transformation with pET22b-CPP-DsRed plasmids, E. coli Rosetta gamiB(DE3)pLysS cells were cultured in 2x YT medium supplemented with $0.4 \%$ glucose and antibiotics until $\mathrm{OD}_{600}$ reached 0.6 . Then, IPTG was adjusted to $1 \mathrm{mM}$ and cells were cultured for another $4 \mathrm{~h}$. The expression level of R11DsRed recombinant protein in the total lysate was nearly undetectable (left panel of Figure 2(a)); therefore, only the other five recombinant proteins were characterized in the following

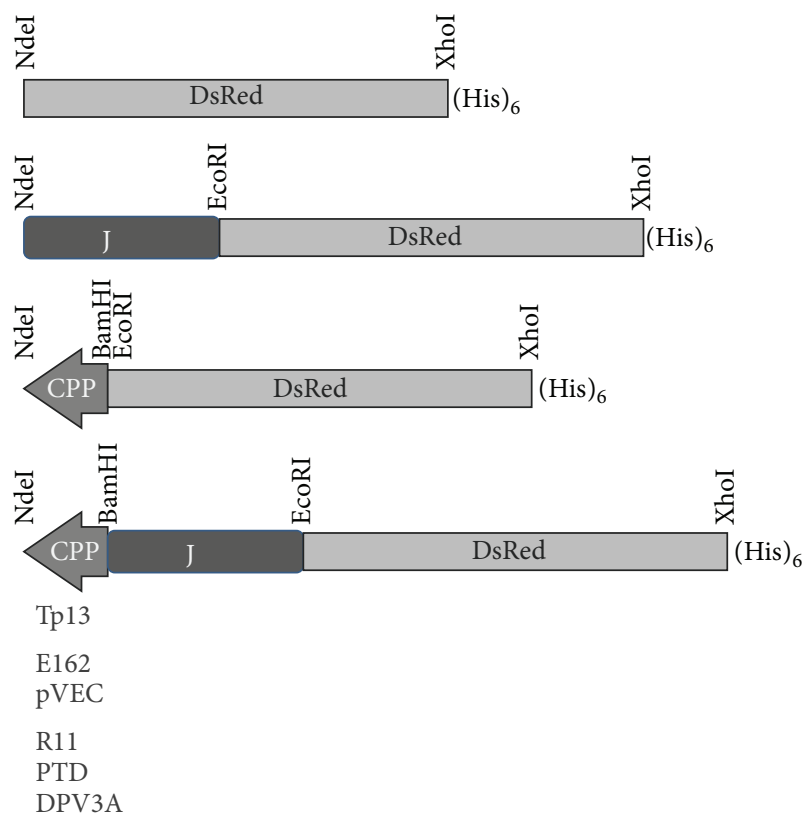

FIgURE 1: Representative structures of the CPP-DsRed and CPP-JDsRed. Oligonucleotide pairs encoding DPV3A, E162, pVEC, R11, and TP13 listed in Table 1 were inserted between NdeI and EcoRI sites of pET22b to create pET22b-DPV3A, pET22b-E162, pET22bpVEC, pET22b-R11, pET22b-PTD, and pET22b-TP13, respectively. The DsRed cDNA fragment cut from pET22b-PTD-J-DsRed by the EcoRI site at $5^{\prime}$-end and the XhoI site at $3^{\prime}$-end was inserted into the above vectors to prepare pET22b-DPV3A-DsRed, pET22b-E162DsRed, pET22b-pVEC-DsRed, pET22b-R11-DsRed, pET22b-PTDDsRed, and pET22b-TP13-DsRed. The J-DsRed cDNA fragment cut from pET22b-PTD-J-DsRed by the BamHI site at $5^{\prime}$-end and the XhoI site at $3^{\prime}$-end was inserted into the above vectors to prepare pET22b-DPV3A-J-DsRed, pET22b-E162-J-DsRed, pET22b-pVECJ-DsRed, pET22b-R11-J-DsRed, and pET22b-TP13-J-DsRed.

steps. After homogenization by ultrasonication, the cell lysate can be separated into soluble (supernatant) and insoluble (pellet) fractions by centrifugation. PTD- and DPV3A-DsRed recombinant proteins were found in the soluble fraction; on the other hand, TP13-, E162-, and pVEC-DsRed recombinant proteins were found in the insoluble fraction (data not shown). The growth rates of the TP13- and E162-DsRed cultures were slower. After removal of cells by centrifugation and $0.22 \mu \mathrm{m}$ membrane filtration, the protein contents in medium after IPTG induction were analyzed by SDS-PAGE. As the patterns shown in the left panel of Figure 2(b), large amounts of cellular proteins of E. coli were detected in the TP13and E162-DsRed lanes; however, a $38 \mathrm{kDa}$ protein band was dominantly detected (indicated by an arrow) in the PTDand DPV3A-DsRed lanes. These results indicated that TP13and E162-DsRed recombinant proteins might disturb the cell membrane of $E$. coli host cells and cause cell disruption, even if most of them were present in the insoluble form.

3.3. Expression of CPP-J-DsRed Recombinant Proteins. The expression characteristics of PTD-J-, DPV3A-J-, TP13-J-, E162-J-, and pVEC-J-DsRed are similar to those of PTD-, 

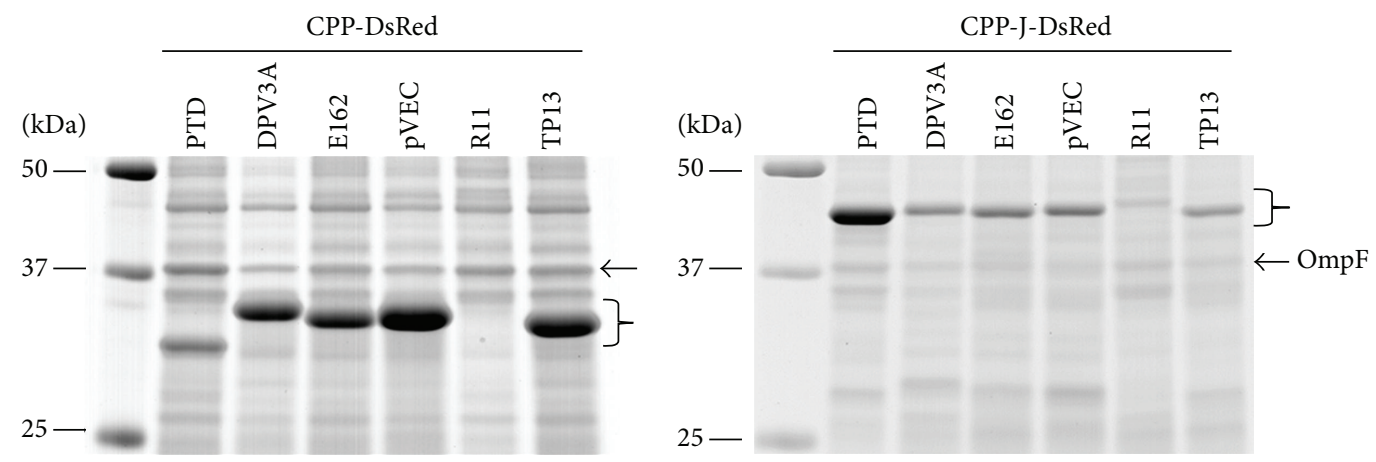

(a)
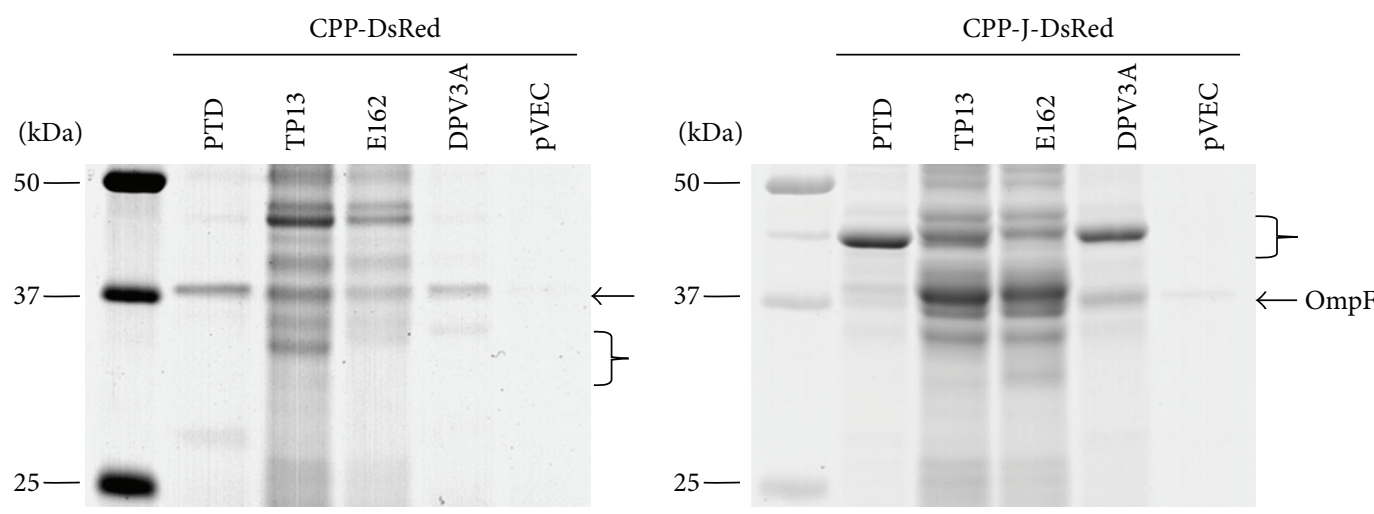

(b)

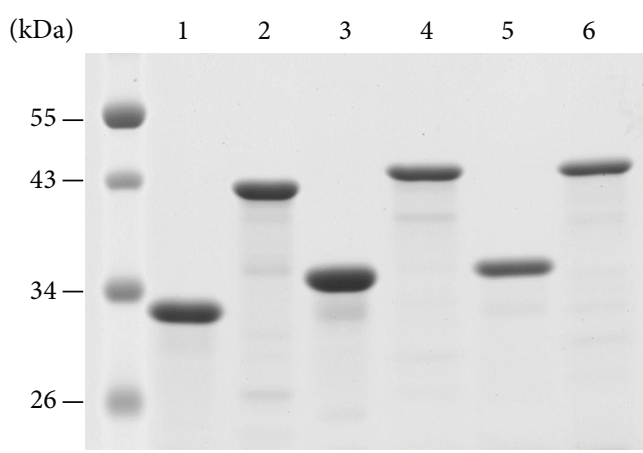
(1) DsRed
(4) PTD-J-DsRed
(2) J-DsRed
(5) DPV3A-DsRed
(3) PTD-DsRed
(6) DPV3A-J-DsRed

(c)

Figure 2: Expression of CPP-DsRed recombinant proteins. (a) Total lysates of E. coli expressing PTD-DsRed, DPV3A-DsRed, pET22b-E162DsRed, pVEC-DsRed, R11-DsRed, and TP13-DsRed recombinant proteins (left panel) as well as those of PTD-J-DsRed, DPV3-J-DsRed, E162J-DsRed, pVEC-J-DsRed, R11-J-DsRed, and TP13-J-DsRed recombinant proteins (right panel) were analyzed by SDS-PAGE. The recombinant proteins are indicated by braces. (b) The protein contents of medium fractions from E. coli expressing PTD-DsRed, DPV3A-DsRed, E162DsRed, pVEC-DsRed, and TP13-DsRed recombinant proteins (left panel) as well as those of PTD-J-DsRed, DPV3-J-DsRed, E162-J-DsRed, pVEC-J-DsRed, R11-J-DsRed, and TP13-J-DsRed (right panel) were analyzed by SDS-PAGE. A $38 \mathrm{kDa}$ common secreted protein OmpF from host cells is indicated by an arrow as internal standard and the recombinant proteins are indicated by braces. (c) The soluble DsRed, J-DsRed, PTD-DsRed, PTD-J-DsRed, DPV3A-DsRed, and DPV3A-J-DsRed recombinant proteins were purified by Ni-Sepharose 6 Fast Flow affinity column chromatography. Each lane was loaded with $20 \mu \mathrm{g}$ of purified recombinant proteins. 


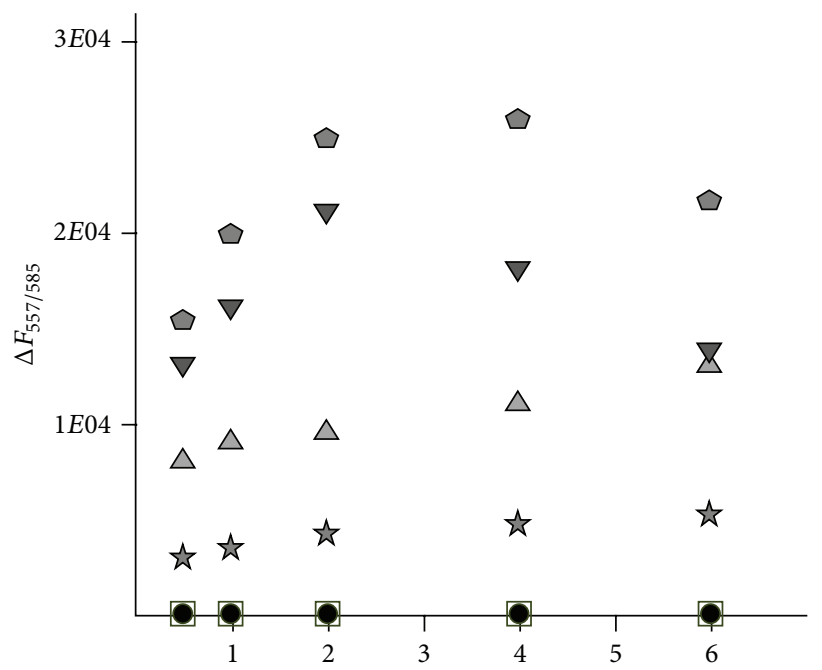

(h)

Huh-7 cells $40 \mu \mathrm{g} / \mathrm{mL}$ $\checkmark$ PTD-J-DsRed $\nabla$ DPV3A-J-DsRed $\triangle$ DPV3A-DsRed

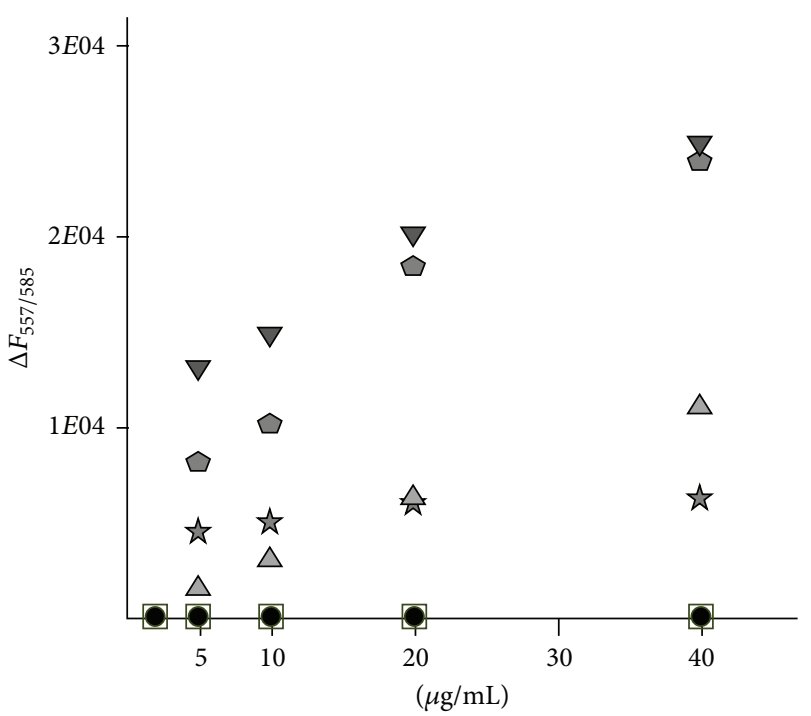

Huh-7 cells

$2 \mathrm{~h}$

$\checkmark$ PTD-J-DsRed

$\nabla$ DPV3A-J-DsRed

$\triangle$ DPV3A-DsRed

(a)

(b)

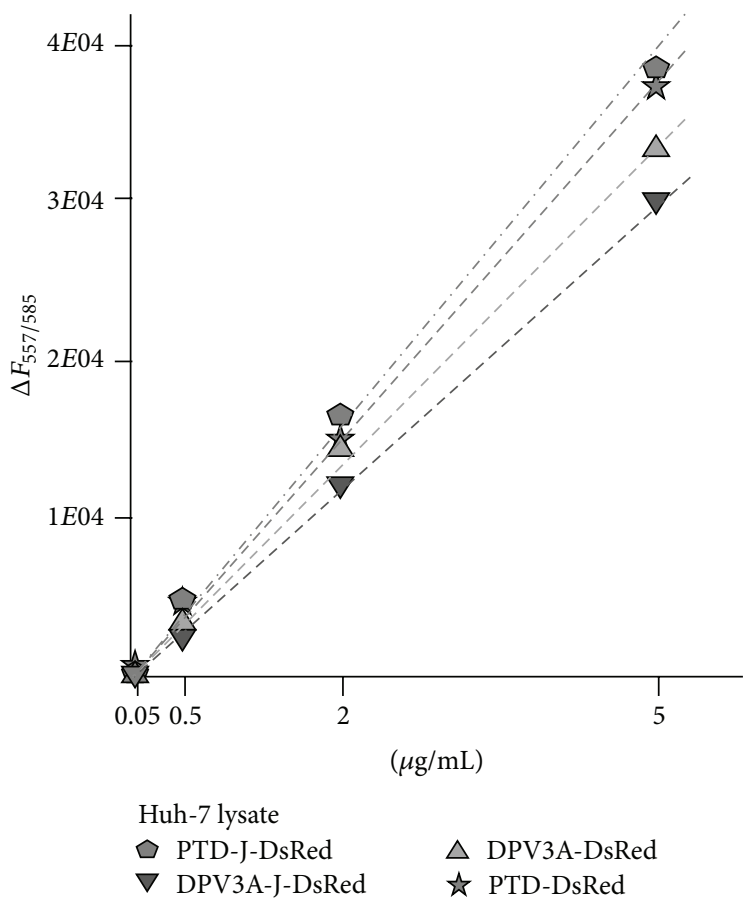

(c)

Figure 3: Transduction of PTD-DsRed, DPV3A-DsRed, PTD-J-DsRed, and DPV3A-J-DsRed recombinant proteins. (a) $1.5 \times 10^{5}$ Huh-7 cells in a well of 24-well plate were incubated with $0.2 \mathrm{~mL}$ of PTD-DsRed, DPV3A-DsRed, PTD-J-DsRed, or DPV3A-J-DsRed recombinant protein $(40 \mu \mathrm{g} / \mathrm{mL})$ for $0.5,1,2,4$, or $6 \mathrm{~h}$. The cells were washed with PBS twice and the recombinant proteins incorporated into cells were released by $0.2 \mathrm{~mL}$ of $1 \%$ Triton X-100/PBS. After centrifugation to remove cell debris, $0.1 \mathrm{~mL}$ of the supernatant was transferred to a well of a 96-well plate and the amounts of recombinant proteins were measured by a fluorometer. The stimulating wave length and emission wave length were set at $557 \mathrm{~nm}$ and $585 \mathrm{~nm}$, respectively. (b) $1.5 \times 10^{5} \mathrm{Huh}-7$ cells in a well of 24 -well plate were incubated with $0.2 \mathrm{~mL} \mathrm{of} 5,10,20$, and $40 \mu \mathrm{g} / \mathrm{mL}$ of the PTD-DsRed, DPV3A-DsRed, PTD-J-DsRed, or DPV3A-J-DsRed recombinant protein for 2 hours. The amounts of recombinant proteins were measured as described before. Experiments were done for four times and the average values were shown. All of the standard deviations were less than 1000. (c) The PTD-DsRed, DPV3A-DsRed, PTD-J-DsRed, and DPV3A-J-DsRed recombinant proteins were diluted in Huh-7 cell lysate to $0.05,0.5,2$, and $5 \mu \mathrm{g} / \mathrm{mL}$ and the fluorescence values were measured as described above. 


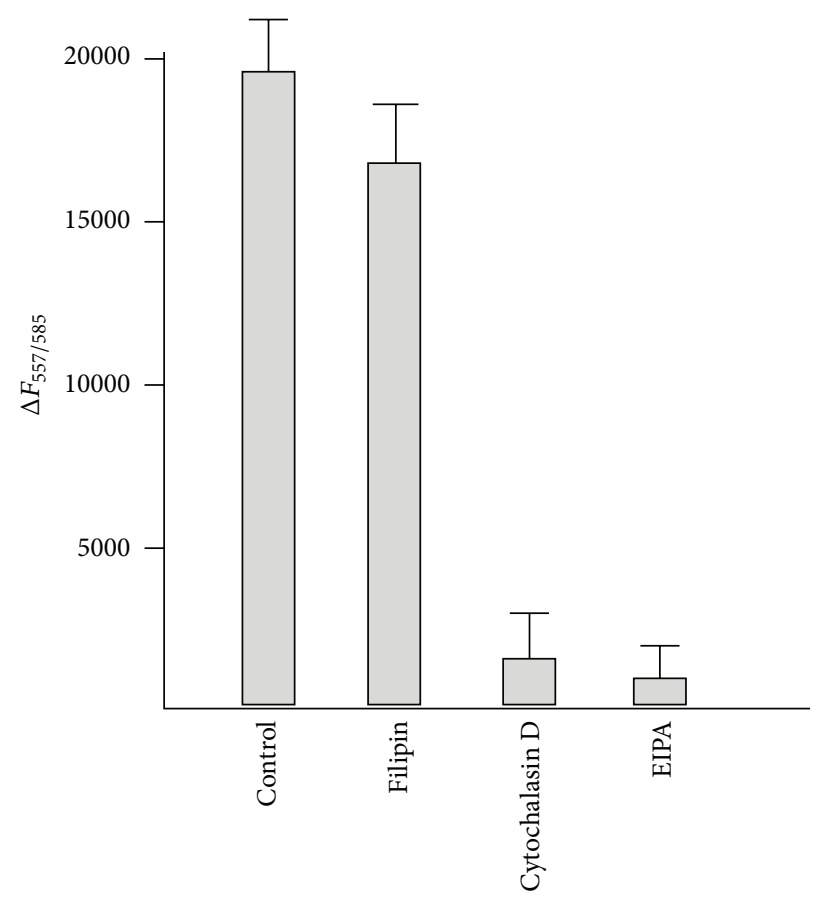

FIGURE 4: Macropinocytosis is involved in the cell-penetration pathway of PTD-J-DsRed. Huh-7 cells were pretreated with filipin $(5 \mu \mathrm{g} / \mathrm{mL})$, EIPA $(100 \mu \mathrm{M})$, or cytochalasin D $(10 \mu \mathrm{M})$ for 1 hour before the treatment of the PTD-J-DsRed recombinant protein $(40 \mu \mathrm{g} / \mathrm{mL})$ for $2 \mathrm{hours}$. The fluorescence values of $100 \mu \mathrm{L}$ cleared lysate samples were measured (three experiments were performed and the $P$ values of filipin, cytochalasin D, and EIPA data relative to the control are 0.0257, 0.000812, and 0.000165, resp.).

DPV3A-, TP13-, E162-, and pVEC-DsRed, respectively (right panel of Figure 2(a)). The major difference is that significant amounts of PTD-J-DsRed and DPV3-J-DsRed recombinant proteins were presented in the medium fractions (right panel of Figure 2(b)). These two "secreted" forms of recombinant proteins could be purified by Ni-NTA affinity column and contain $\mathrm{N}$-terminal amino acid sequences the same as those of PTD-J-DsRed and DPV3A-J-DsRed indicating that they have intact $\mathrm{N}$ - and $\mathrm{C}$-termini. In comparison with the amount of recombinant proteins that remain within cells, about $10 \%$ of these two recombinant proteins were in the secreted fractions.

3.4. Cell-Penetrating Activities of PTD-, DPV3A-, PTD-J-, and DPV3A-J-DsRed Recombinant Proteins. Recombinant DsRed, J-DsRed, PTD-DsRed, PTD-J-DsRed, DPV3ADsRed, and DPV3A-J-DsRed proteins (Figure 2(c)) were purified from cell lysates by Ni-NTA-Sepharose affinity column chromatography. After elution by $250 \mathrm{mM}$ imidazole, these proteins were dialyzed against 100 volumes of PBS twice, and aliquots were stored at $-80^{\circ} \mathrm{C}$.

The cell-penetrating activities of the four recombinant proteins were tested. At first, the time course of recombinant protein transduction to Huh-7 cells was measured at $0.51,2$, 4 , and 6 hours using $40 \mu \mathrm{g} / \mathrm{mL}$ of each recombinant protein in serum-free Opti-MEM medium. Both of the DsRed and J-DsRed could not penetrate into Huh-7 cells. The amount of the PTD-DsRed and DPV3A-DsRed proteins transduced increased with time up to 6 hours. However, the time for optimal amount of PTD-J-DsRed and DPV3A-J-DsRed transduced into cells was around 2 to 4 hours (Figure 3(a)). Then, Huh-7 cells were treated with $5,10,20$, and $40 \mu \mathrm{g} / \mathrm{mL}$ of each recombinant protein in the same medium for 2 hours. The amounts of recombinant proteins incorporated increased roughly proportional to the amounts of the recombinant proteins added (Figure 3(b)). To estimate the amounts of recombinant proteins transduced into cells, calibration curves of the fluorescence values of $100 \mu \mathrm{L}$ sample per well relative to the concentrations of recombinant proteins in total cell lysate were illustrated in Figure 3(c). According to the calibration curves, about $2 \%$ of PTD-DeRed and DPV3A-DsRed and 7\% of PTD-J-DeRed and DPV3A-J-DsRed can transduce into cells when $40 \mu \mathrm{g} / \mathrm{mL}$ of recombinant proteins was used.

It had been suggested that macropinocytosis was involved in the penetration pathway of arginine-rich CPPs $[19,20]$. The entrance route of PTD-J-DeRed was analyzed by using inhibitors of different endocytic pathways [26]. The EIPA and cytochalasin D (both are inhibitors on macropinocytosis) and filipin which is an inhibitor of caveolae-mediated endocytosis were tested. As shown in Figure 4, only EIPA and cytochalasin $\mathrm{D}$ could reduce the amount of intracellular PTD-J-DsRed indicating that macropinocytosis was involved in its entrance. The same result was obtained for DPV3A-J-DsRed (data not shown). The pattern of PTD-DsRed and PTD-J-DsRed recombinant proteins incorporated into Huh-7 cells was further analyzed using immunofluorescence microscopy. More 


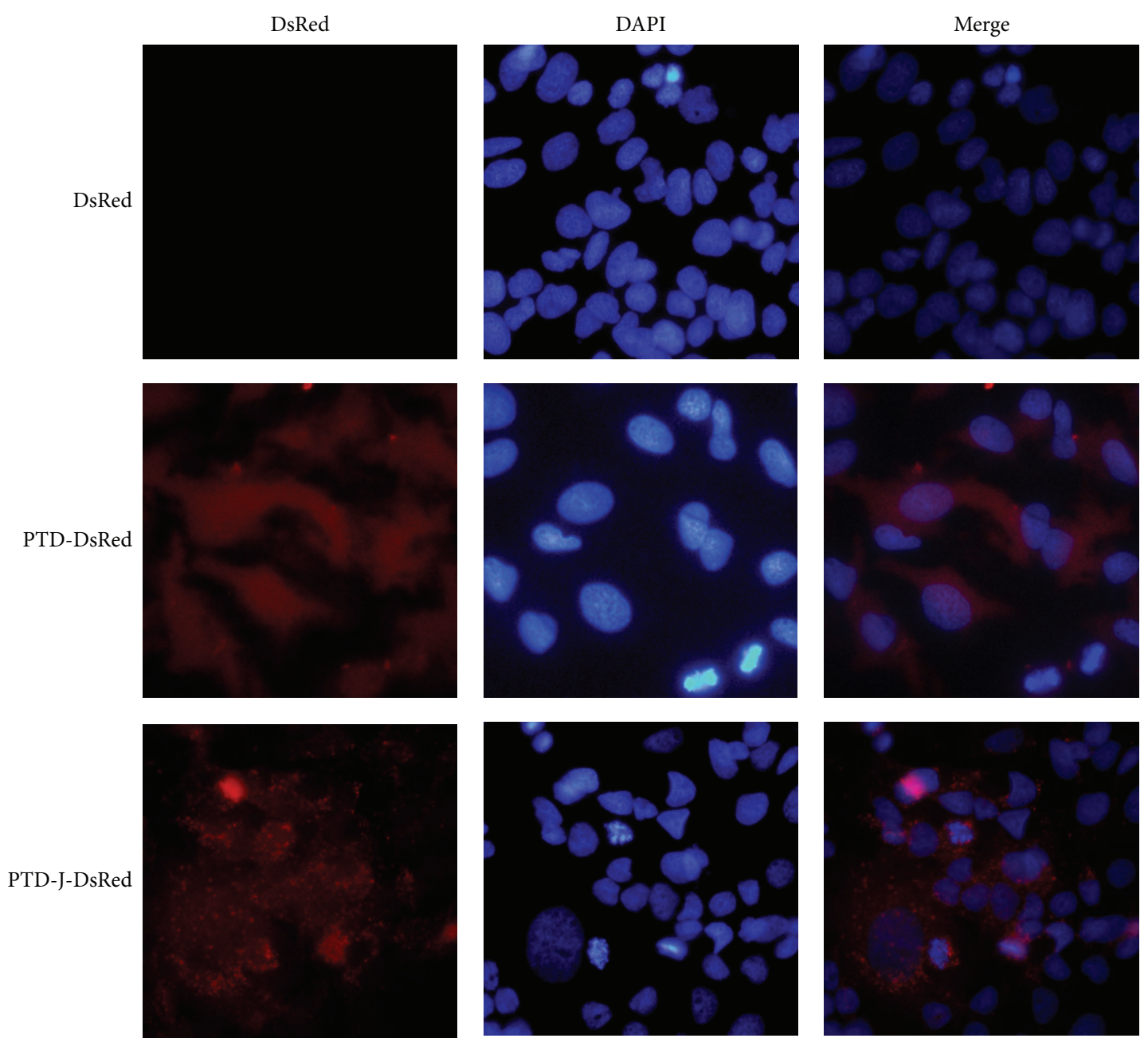

FIGURE 5: Fluorescence microscopic analysis of the cellular uptake of DsRed, PTD-DsRed, and PTD-J-DsRed recombinant proteins. Huh-7 cells were treated with $40 \mu \mathrm{g} / \mathrm{mL}$ of PTD-DsRed or PTD-J-DsRed recombinant protein for $2 \mathrm{~h}$. After two PBS washes, cells were fixed with $4 \%$ paraformaldehyde/PBS for $10 \mathrm{~min}$ at room temperature, counterstained with DAPI, and observed under a fluoromicroscope.

granular signals were observed in the PTD-J-DsRed image (Figure 5).

\section{Discussion}

\subsection{PTD-J-DsRed and DPV3A-J-DsRed Recombinant Proteins} Were Found in the Medium. During the expression of PTD-JDsRed and DPV3A-J-DsRed recombinant proteins by E. coli, about $10 \%$ of total recombinant proteins could be isolated from medium fraction (Figure 2(b)). These "secreted" forms of recombinant proteins were purified by Ni-NTA affinity column and determined with intact $\mathrm{N}$-termini by amino acid sequencing indicating that they are the same as the cellular forms. It is interesting to distinguish how PTD-J-DsRed and DPV3A-J-DsRed recombinant proteins can be released from E. coli cells into the medium. Proteins located outside the inner membrane of $E$. coli are usually synthesized with $\mathrm{N}$ terminal signal peptides to target them to either $\operatorname{Sec}[27,28]$ or Tat (twin-arginine translocation) $[29,30]$ protein export pathway. The major difference between these two export systems is that the Sec apparatus translocates unfolded polypeptides across the membrane, whereas the Tat complex transports already folded proteins. The Tat pathway can transport a heterooligomeric protein complex in which only one subunit possesses a Tat-targeting signal peptide through membrane at once. For example, only the small HybO subunit of HybOC hydrogenase 2 complex has the Tat-targeting signal peptide. The large HybC subunit was transported in complex with the HybO subunit [31]. Another case is the SoxYZ protein complex involved in thiosulfate oxidation. Only SoxY has a Tattargeting signal peptide and SoxZ is exported in complex with SoxY [29]. Although there is not any datum to support that PTD-J-DsRed or DPV3A-J-DsRed could be carried across cellular membrane by an unknown protein with Tat-targeting signal peptide, it provides a possible pathway to interpret how a protein without signal peptide can be transported across the inner membrane of $E$. coli. 
4.2. PTD-J-DsRed and DPV3A-J-DsRed Recombinant Proteins Transduce More Effectively Than Their PTD-DsRed and DPV3A-DsRed Counterparts. When Huh-7 cells were treated with the same concentration $(40 \mu \mathrm{g} / \mathrm{mL})$ of recombinant proteins, the amounts of PTD-DsRed and DPV3A-DsRed incorporated were slightly increased with time after 1 hour. However, the maximal amounts of PTD-J-DsRed and DPV3A-JDsRed within cells were detected around 2 to 4 hours. Then, the fluorescence values decreased because cells began to be lysed (Figure 3(a)). DsRed and J-DsRed could not penetrate into Huh-7 cells. PTD-J-DsRed and DPV3A-J-DsRed could penetrate into Huh-7 cells more effectively than PTD-DsRed and DPV3A-DsRed, respectively, did. These results indicate that the J-domain itself has no cell-penetrating ability; however, it can enhance the cell-penetrating activity of PTD and DPV3A. When Huh-7 cells were treated with $40 \mu \mathrm{g} / \mathrm{mL}$ of PTD-J-DsRed for 2 hours, about $0.6 \mu \mathrm{g}$ of recombinant proteins was penetrated into $10^{4}$ cells, corresponding to $10^{9}$ molecules per cell.

The pattern of PTD-DsRed and PTD-J-DsRed recombinant proteins incorporated into Huh-7 cells was further analyzed using immunofluorescence microscopy. More granular signals were observed in the PTD-J-DsRed image (Figure 5). In addition, the penetration of PTD-J-DsRed recombinant protein into Huh-7 cells was inhibited by EIPA and cytochalasin D (Figure 4). These phenomena indicate that endocytic uptake-endosomal escape pathway may be the major route for PTD-J-DsRed to penetrate into Huh-7 cells. In conclusion, the J-domain can assist CPPs and their cargo to penetrate through the cellular membrane of both E. coli and Huh-7 cell more effectively.

\section{Conclusion}

By using red fluorescence protein DsRed as a reporter, the recombinant proteins PTD-J-DsRed and DPV3A-J-DsRed performed higher cell-penetrating activity than PTD-DsRed and DPV3A-DsRed, respectively. Because both DsRed and JDsRed recombinant proteins could not penetrate into cells, it is suggested that the J-domain could help cell-penetrating peptides PTD and DPV3A as well as their cargo to penetrate through the cellular membrane more effectively.

\section{Conflict of Interests}

The authors declare that there is no conflict of interests regarding the publication of this paper.

\section{Acknowledgments}

This work was supported by the Grants NSC 102-2320-B-059001-MY3, NSC 101-2622-B-059-001-CC2, and NSC 99-2313B-059-004-MY3 from National Science Council and 101-EC17-A-02-04-0454 from Ministry of Economics, Taiwan, to Chin-Kai Chuang.

\section{References}

[1] A. D. Frankel and C. O. Pabo, "Cellular uptake of the tat protein from human immunodeficiency virus," Cell, vol. 55, no. 6, pp. 1189-1193, 1988.

[2] S. Fawell, J. Seery, Y. Daikh et al., "Tat-mediated delivery of heterologous proteins into cells," Proceedings of the National Academy of Sciences of the United States of America, vol. 91, no. 2, pp. 664-668, 1994.

[3] E. Vivès, P. Brodin, and B. Lebleu, "A truncated HIV-1 Tat protein basic domain rapidly translocates through the plasma membrane and accumulates in the cell nucleus," The Journal of Biological Chemistry, vol. 272, no. 25, pp. 16010-16017, 1997.

[4] H. Nagahara, A. M. Vocero-Akbani, E. L. Snyder et al., "Transduction of full-length TAT fusion proteins into mammalian cells: TAT-p $27^{\text {Kipl }}$ induces cell migration," Nature Medicine, vol. 4, no. 12, pp. 1449-1452, 1998.

[5] S. R. Schwarze, A. Ho, A. Vocero-Akbani, and S. F. Dowdy, "In vivo protein transduction: delivery of a biologically active protein into the mouse," Science, vol. 285, no. 5433, pp. 1569$1572,1999$.

[6] S. R. Schwarze and S. F. Dowdy, "In vivo protein transduction: intracellular delivery of biologically active proteins, compounds and DNA," Trends in Pharmacological Sciences, vol. 21, no. 2, pp. 45-48, 2000.

[7] D. Derossi, A. H. Joliot, G. Chassaing, and A. Prochiantz, "The third helix of the Antennapedia homeodomain translocates through biological membranes," The Journal of Biological Chemistry, vol. 269, no. 14, pp. 10444-10450, 1994.

[8] F. Milletti, "Cell-penetrating peptides: classes, origin, and current landscape," Drug Discovery Today, vol. 17, no. 15-16, pp. 850-860, 2012.

[9] A. Gautam, H. Singh, A. Tyagi et al., "CPPsite: a curated database of cell penetrating peptides," Database, vol. 2012, Article ID bas015, 2012.

[10] A. Gräslund, F. Madani, S. Lindberg, Ü. Langel, and S. Futaki, "Mechanisms of cellular uptake of cell-penetrating peptides," Journal of Biophysics, vol. 2011, Article ID 414729, 10 pages, 2011.

[11] M. Pooga, M. Hällbrink, M. Zorko, and Ü. Langel, "Cell penetration by transportan," FASEB Journal, vol. 12, no. 1, pp. 67-77, 1998.

[12] U. Soomets, M. Lindgren, X. Gallet et al., "Deletion analogues of transportan," Biochimica et Biophysica Acta (BBA)_Biomembranes, vol. 1467, no. 1, pp. 165-176, 2000.

[13] A. Elmquist, M. Lindgren, T. Bartfai, and Ü. Langel, "Vecadherin-derived cell-penetrating peptide, pVEC with carrier functions," Experimental Cell Research, vol. 269, no. 2, pp. 237244, 2001.

[14] M. Hällbrink, K. Kilk, A. Elmquist et al., "Prediction of cellpenetrating peptides," International Journal of Peptide Research and Therapeutics, vol. 11, no. 4, pp. 249-259, 2005.

[15] T. Takeuchi, M. Kosuge, A. Tadokoro et al., "Direct and rapid cytosolic delivery using cell-penetrating peptides mediated by pyrenebutyrate," ACS Chemical Biology, vol. 1, no. 5, pp. 299303, 2006.

[16] C. de Coupade, A. Fittipaldi, V. Chagnas et al., "Novel humanderived cell-penetrating peptides for specific subcellular delivery of therapeutic biomolecules," Biochemical Journal, vol. 390, no. 2, pp. 407-418, 2005.

[17] I. Nakase, M. Niwa, T. Takeuchi et al., "Cellular uptake of arginine-rich peptides: roles for macropinocytosis and actin 
rearrangement," Molecular Therapy, vol. 10, no. 6, pp. 1011-1022, 2004.

[18] P. Guterstam, F. Madani, H. Hirose et al., "Elucidating cellpenetrating peptide mechanisms of action for membrane interaction, cellular uptake, and translocation utilizing the hydrophobic counter-anion pyrenebutyrate," Biochimica et Biophysica Acta (BBA)-Biomembranes, vol. 1788, no. 12, pp. 2509-2517, 2009.

[19] I. M. Kaplan, J. S. Wadia, and S. F. Dowdy, "Cationic TAT peptide transduction domain enters cells by macropinocytosis," Journal of Controlled Release, vol. 102, no. 1, pp. 247-253, 2005.

[20] I. Nakase, A. Tadokoro, N. Kawabata et al., "Interaction of arginine-rich peptides with membrane-associated proteoglycans is crucial for induction of actin organization and macropinocytosis," Biochemistry, vol. 46, no. 2, pp. 492-501, 2007.

[21] A. Ziegler and J. Seelig, "Interaction of the protein transduction domain of HIV-1 TAT with heparan sulfate: binding mechanism and thermodynamic parameters," Biophysical Journal, vol. 86, no. 1, pp. 254-263, 2004.

[22] C.-K. Chuang, Y.-S. Su, C.-T. Fan, W.-C. Lee, and M.-Y. Chen, "A dual-functional E. coli vector for expressing recombinant protein with high solubility and antigen presentation ability," Protein Expression and Purification, vol. 65, no. 1, pp. 51-56, 2009.

[23] T. Y. Lin, Y. S. Su, L. Y. Hung, and C. K. Chuang, "Hsp70 protein can enhance the immunogenicity of FMDV VP1 epitopes fused with PTD-J domain," Scholarly Journal of Biological Science, vol. 2, no. 4, pp. 39-48, 2013.

[24] W. S. Sanders, C. I. Johnston, S. M. Bridges, S. C. Burgess, and K. O. Willeford, "Prediction of cell penetrating peptides by support vector machines," PLoS Computational Biology, vol. 7, no. 7, Article ID e1002101, 2011.

[25] D. Kim, C.-H. Kim, J.-I. Moon et al., "Generation of human induced pluripotent stem cells by direct delivery of reprogramming proteins," Cell Stem Cell, vol. 4, no. 6, pp. 472-476, 2009.

[26] A. El-Sayed and H. Harashima, "Endocytosis of gene delivery vectors: from clathrin-dependent to lipid raft-mediated endocytosis," Molecular Therapy, vol. 21, no. 6, pp. 1118-1130, 2013.

[27] J. A. Lycklama a Nijeholt and A. J. M. Driessen, "The bacterial Sec-translocase: structure and mechanism," Philosophical Transactions of the Royal Society B: Biological Sciences, vol. 367, no. 1592, pp. 1016-1028, 2012.

[28] D. J. F. du Plessis, N. Nouwen, and A. J. M. Driessen, "The Sec translocase," Biochimica et Biophysica Acta-Biomembranes, vol. 1808, no. 3, pp. 851-865, 2011.

[29] T. Palmer and B. C. Berks, "The twin-arginine translocation (Tat) protein export pathway," Nature Reviews Microbiology, vol. 10, no. 7, pp. 483-496, 2012.

[30] C. Robinson, C. F. R. O. Matos, D. Beck et al., "Transport and proofreading of proteins by the twin-arginine translocation (Tat) system in bacteria," Biochimica et Biophysica Acta, vol. 1808, no. 3, pp. 876-884, 2011.

[31] A. Rodrigue, A. Chanal, K. Beck, M. Müller, and L.-F. Wu, "Cotranslocation of a periplasmic enzyme complex by a hitchhiker mechanism through the bacterial tat pathway," The Journal of Biological Chemistry, vol. 274, no. 19, pp. 13223-13228, 1999. 

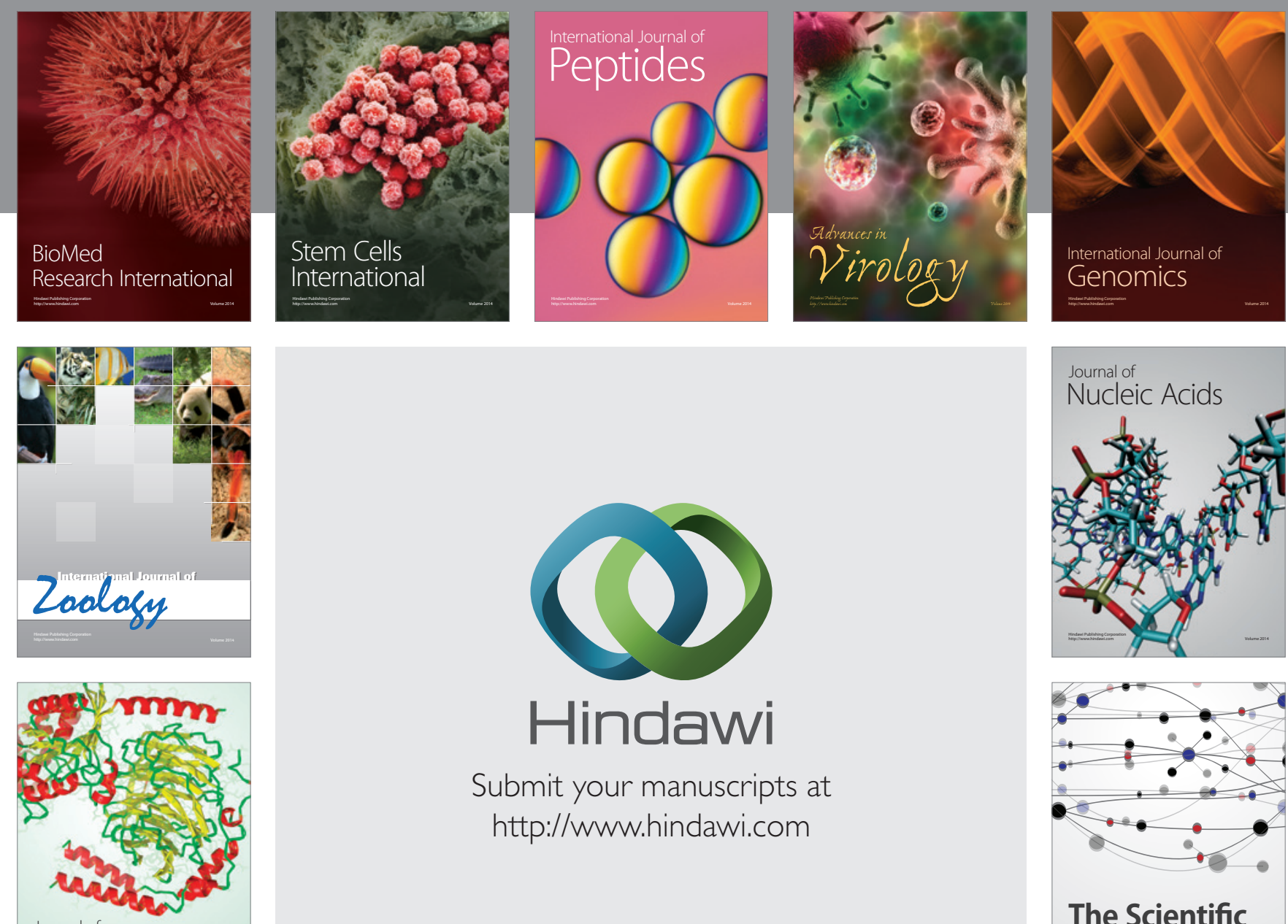

Submit your manuscripts at

http://www.hindawi.com

Journal of
Signal Transduction
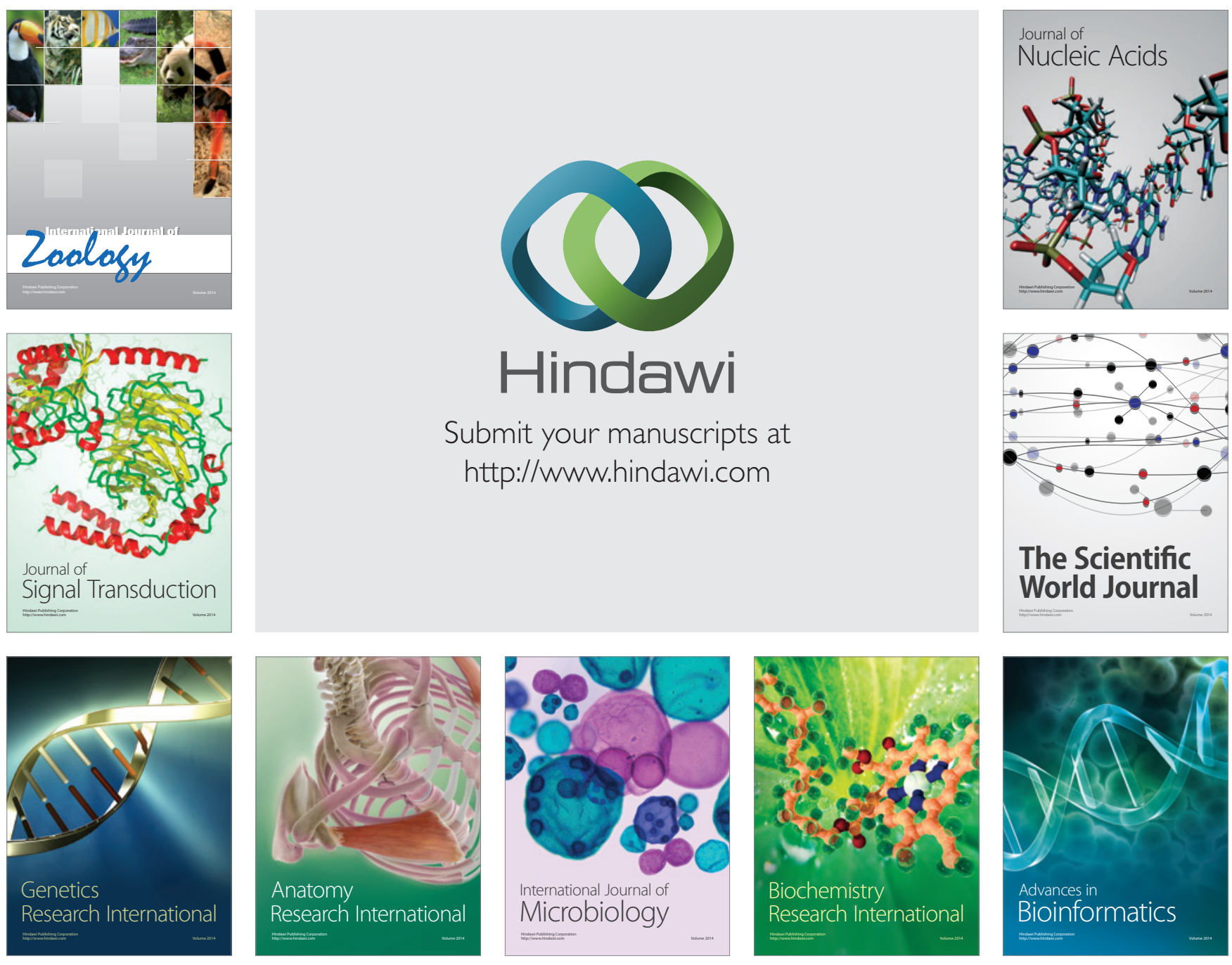

The Scientific World Journal
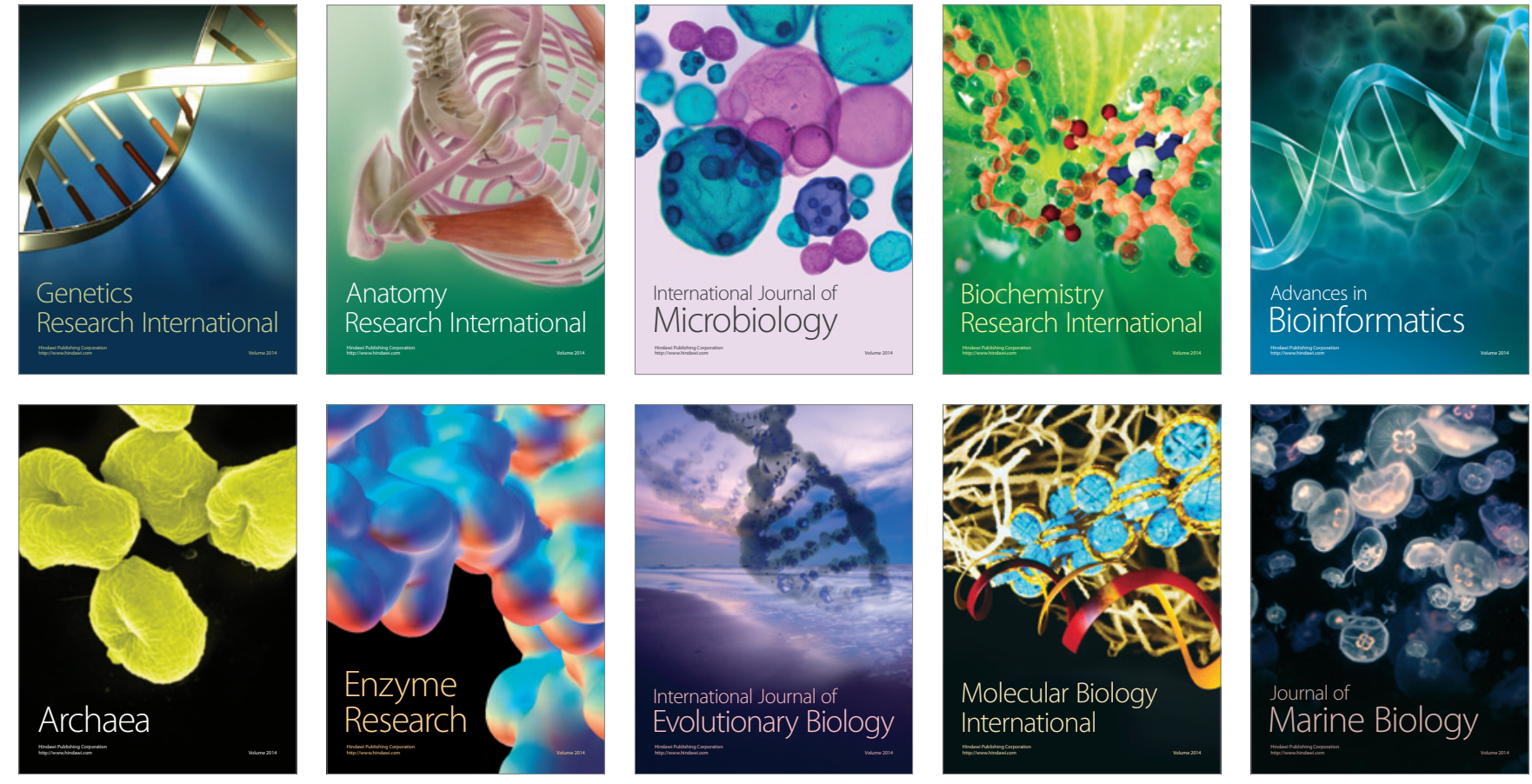